\title{
COMPORTAMENTO DA LINHA DO SOLO OBTIDA POR ESPECTRORRADIOMETRIA LABORATORIAL PARA DIFERENTES CLASSES DE SOLO ${ }^{(1)}$
}

\author{
Marcos Rafael Nanni ${ }^{(2)}$ \& José Alexandre Melo Demattê $\hat{e}^{(3)}$
}

\begin{abstract}
RESUMO
Com vistas em avaliar o comportamento da linha do solo para diferentes classes de solos, este trabalho foi realizado, utilizando-se dados de reflectância por meio de um espectrorradiômetro em laboratório. Amostras representativas dos horizontes superficiais e subsuperficiais de 18 classes coletadas em uma área piloto no Município de Rafard, sudoeste de São Paulo, foram secas, peneiradas e acondicionadas para leitura espectral. Os dados foram tabulados, para simularem as bandas 3 e 4 do TM-Landsat e construção dos gráficos. Os resultados demonstraram que as linhas de solo obtidas para as amostras de ambas as camadas comportaram-se de maneira semelhante, estando dispostas ortogonalmente aos eixos de um gráfico, com valores de $R^{2}$ próximos a 1,0 para todas as classes avaliadas. Não se observou uma única linha de solo, embora os dados revelem que cada solo apresenta uma linha individual e característica. De modo complementar, destacouse a utilização deste índice como método auxiliar na discriminação de classes de solos, uma vez que os solos com textura arenosa e com menores teores de Fe total discriminaram-se daqueles mais argilosos e com maiores teores de Fe, além de apresentarem valores de reflectância mais elevados. Por fim, constatou-se que a matéria orgânica não constituiu fator determinante no comportamento espectral em relação à linha do solo.
\end{abstract}

Termos de indexação: Reflectância, índice de vegetação, comportamento espectral.

(1) Parte da Tese de Doutorado do primeiro autor, apresentada ao Departamento de Solos e Nutrição de Plantas Escola Superior de Agricultura "Luiz de Queiroz" - ESALQ-USP. Recebido para publicação em julho de 2005 e a provado em outubro de 2006.

${ }^{(2)}$ Professor do Departamento de Agronomia, Universidade Estadual de Maringá - UEM. Av. Colombo 5790, CEP $87020-900$ Maringá (PR). E-mail: mrnanni@uem.br

(3) Professor do Departamento de Solos e Nutrição de Plantas, Escola Superior de Agricultura "Luiz de Queiroz" - ESALQ, Av. Pádua Dias 11, Caixa Postal 9, CEP 13418-900 Piracicaba (SP). E-mail: jamdemat@esalq.usp.br 


\title{
SUMMARY: SOIL LINE BEHAVIOR OBTAINED BY LABORATORIAL SPECTRORADIOMETRY FOR DIFFERENT SOIL CLASSES
}

\begin{abstract}
The aim of this study was to evaluate the behavior of the soil line for different soil classes by using reflectance data with a spectroradiometer in the laboratory. Representative samples of the surface and subsurface horizons of 18 soil classes collected in a pilot area in Rafard county, southwest São Paulo state, were dried, sieved and accomodated for spectral reading. Data were computed in order to simulate TM-Landsat 3 and 4 bands and to draw the graphs. The findings showed that the soil lines obtained for the samples of both soil layers behave similarly and are distributed orthogonal to the graph axes with $R^{2}$ values at about 1.0 for all classes evaluated. It was observed that there is not a unique soil line, and the data suggest that each soil has an individual line and features. In addition, the use of such an index can be an auxiliary method for discriminating soil classes, since sandy soils, with a lower iron content were different from the more clayey ones with a higher iron content, besides presenting higher reflectance values. Finally, it was observed that the organic matter was not a determinant factor regarding the spectral behavior in relation to the soil line.
\end{abstract}

Index terms: Reflectance, vegetation index, spectral behavior, iron, clay.

\section{INTRODUÇÃO}

A reflectância dos solos é governada por diversos fatores. As propriedades químicas influenciam sua assinatura espectral pelos processos de absorção. A variação espacial e temporal dos solos ou da vegetação é quase que imprevisível. Correções dos dados de sensoriamento remoto são, portanto, necessárias para sua correta avaliação. Para tanto, utilizam-se, em sensoriamento remoto, técnicas de processamento digital de imagens com o objetivo de melhorar o aspecto visual de certas feições estruturais para o analista humano e fornecer outros subsídios para a sua interpretação, gerando até mesmo produtos que possam ser submetidos posteriormente a outros processamentos (INPE, 1999).

Essas técnicas envolvem uma série de operações matemáticas cuja finalidade é a produção de imagens que destaquem o objeto ou alvo de estudo. Nestas operações, utiliza-se uma (ou duas) banda de uma mesma área geográfica, previamente georreferenciada. Realiza-se a operação “pixel” a "pixeP”, por meio de uma regra matemática definida, tendo como resultado uma banda que representa a combinação das bandas originais. Tais operações permitem a compressão de dados, diminuindo o número de bandas, ocasionando, contudo, perda da informação original.

De maneira geral, utilizam-se as seguintes operações para obter informações adicionais de uma cena orbital ou realçar diferenças e semelhanças espectrais: soma ou adição, subtração, multiplicação e divisão entre bandas.

Dentre estas, a divisão ou razão entre bandas numa operação não-linear é utilizada para realçar as diferenças espectrais de um par de bandas, caracterizando determinadas feições da curva de assinatura espectral de alguns alvos.

Para aumentar o contraste entre solo e vegetação, pode-se utilizar a razão entre bandas referentes ao vermelho e infravermelho próximo, constituindo, assim, os chamados índices de vegetação (IV). Geralmente, estes índices referem-se a uma combinação da reflectância de bandas e podem fornecer medidas efetivas da biomassa fotossinteticamente ativa (Kauth \& Thomas, 1976; Jackson, 1983).

Um dos índices de vegetação mais utilizados denomina-se índice de vegetação de diferença normalizada (IVDN), que é obtido dividindo-se a diferença entre a medida de reflectância referente ao intervalo do espectro-eletromagnético, que compreende o infravermelho (IV) e o vermelho (V), e a soma entre esses dois intervalos, ou seja: IVDN $=(\mathrm{IV}-\mathrm{V}) /(\mathrm{IV}+$ V). Segundo Galvão et al. (1999), para o sensor TM do satélite Landsat 5, o vermelho e o infravermelho referem-se aos intervalos de 630 a $690 \mathrm{~nm}$ e 760 a $900 \mathrm{~nm}$, respectivamente, sendo a banda centrada em 660 e $830 \mathrm{~nm}$ para os dois intervalos.

Alguns dos índices de vegetação baseiam-se no fato de que solos expostos num gráfico bi-dimensional, formado por uma banda no visível versus uma banda no infravermelho próximo, ocorrem aproximadamente numa linha denominada "linha do solo". A quantidade de vegetação na cena estudada será, então, proporcional à distância ortogonal euclidiana, no supra-referido gráfico bi-dimensional, entre os pontos de vegetação e a "linha do solo" (Huete, 1989). Reciprocamente, a distância euclidiana baseada nos índices de vegetação tem seu índice ortogonal complementar que está relacionado com as propriedades óticas dos solos para baixas quantidades de vegetação (Fukuhara et al., 1979). 
Uma das maneiras de entender as variações nos dados espectrais em nível orbital é conhecer, inicialmente, os solos pela radiometria em laboratório, que reflete a realidade mais próxima do objeto de estudo (Demattê, 1999).

Para tanto, Demattê et al. (2000) e Demattê \& Nanni (2003) têm utilizado a simulação entre os dados orbitais e terrestres para posterior comparação. Aliás, este procedimento é muito comum, quando se pretende simular como seriam os dados de um sensor orbital (Latz et al., 1984).

Com o objetivo de estabelecer a denominada linha do solo por meio da resposta espectral laboratorial, uma área de grande interesse agrícola no interior do Estado de São Paulo foi utilizada para obtenção de dados espectrais de laboratório, visando à avaliação $\mathrm{e}$ análise da relação entre bandas e, então, caracterizar o seu comportamento espectral.

\section{MATERIAL E MÉTODOS}

A área de estudo está localizada ao sudoeste do Estado de São Paulo, delimitada pelas coordenadas geográficas $23^{\circ} 0$ ' $31,37^{\prime \prime}-22^{\circ} 58^{\prime} 53,97$ " latitude sul e $53^{\circ} 39^{\prime} 47,81^{\prime \prime}-53^{\circ} 37^{\prime} 25,65^{\prime \prime}$ longitude oeste, na região denominada depressão paleozóica (IPT, 1981). Fazendo parte da bacia hidrográfica do Rio Tietê, essa área encontra-se margeada pelo Rio Capivari, próximo ao município de Rafard. O trabalho foi desenvolvido em 185 ha, num total de, aproximadamente, 198 ha, utilizados com a cultura da cana-de-açúcar.
Geologicamente, a área situa-se sobre a Formação Itararé, pertencente ao Grupo Tubarão. Esta formação apresenta litologias predominantes areníticas, de granulação heterogênea, com presença de arenitos feldspáticos e arcósios. Argilitos e folhelhos de colorações variadas, desde o cinza-claro ao escuro, complementam a litologia desta formação e são de ocorrência freqüente. A região revela elementos eruptivos da Formação Serra Geral, compreendendo corpos intrusivos de mesma composição que os basaltos toleíticos (Nanni et al., 2004).

A área apresenta, conforme Nanni (2000), 18 classes de solos (Quadro 1).

Para a coleta de amostras, com vistas em avaliar a resposta espectral no laboratório, foram definidos, para a área de estudo, 185 pontos de amostragem, compreendendo uma grade regular com arestas de $100 \mathrm{~m}$. Para tanto, cada ponto foi devidamente demarcado com o uso de um distanciômetro com feixes de infravermelho e sistemas de prismas refletores. Cada ponto definido para amostragem foi sinalizado com uma estaca de madeira, donde foram obtidas as coordenadas geográficas por meio de sistema de posicionamento global (GPS) e, posteriormente, corrigidas pelo sistema DGPS (Diferential Global Positioning System), para maior acurácia da localização geográfica.

Amostras de solo foram coletadas em cada ponto, em duas profundidades, representativas dos horizontes diagnósticos de superfície e subsuperfície para cada classe presente na paisagem.

Enviadas ao laboratório, as amostras foram secas em estufa de circulação forçada a $45^{\circ} \mathrm{C}$ e tamizadas

\section{Quadro 1. Classes de solos presentes na área de estudo e distribuição quantitativa}

\begin{tabular}{|c|c|c|c|}
\hline $\begin{array}{l}\text { Símbolo da } \\
\text { classe }\end{array}$ & \multicolumn{2}{|c|}{ Área } & Classificação dos solos (Embrapa, 1999) \\
\hline & ha & $\%$ & \\
\hline PVAd & 7,6 & 4,1 & Argissolos Vermelho-Amarelos distróficos típicos textura arenosa/média \\
\hline PVAe1 & 26,7 & 14,3 & Argissolos Vermelho-Amarelos eutróficos típicos textura arenosa/média \\
\hline PVAe2 & 20,2 & 10,8 & Argissolos Vermelho-Amarelos eutróficos típicos textura média/argilosa \\
\hline PVAe3 & 3,6 & 1,9 & Argissolos Vermelho-Amarelos eutróficos abrúpticos textura média/muito argilosa \\
\hline CXd1 & 6,4 & 3,4 & Cambissolos Háplicos Tb alumínicos típicos textura argilosa \\
\hline $\mathrm{CXd} 2$ & 13,6 & 7,3 & Cambissolos Háplicos Tb distróficos típicos textura média \\
\hline CXe1 & 5,2 & 2,7 & Cambissolos Háplicos Tb eutróficos típicos textura argilosa \\
\hline $\mathrm{CXe} 2$ & 9,0 & 4,8 & Cambissolos Háplicos Ta eutróficos lépticos textura argilosa \\
\hline CXe3 & 2,8 & 1,5 & Cambissolos Háplicos Tb eutróficos típicos textura argilosa \\
\hline MTfr & 12,8 & 6,8 & Chernossolos Argilúvicos férricos saprolíticos textura argilosa \\
\hline $\mathrm{LVe}$ & 19,0 & 10,2 & Latossolos Vermelhos eutróficos típicos textura argilosa \\
\hline LVAd & 6,6 & 3,5 & Latossolos Vermelho-Amarelos distróficos típicos textura média \\
\hline $\mathrm{LVAe}$ & 14,0 & 7,5 & Latossolos Vermelho-Amarelos eutróficos típicos textura argilosa \\
\hline RLe1 & 1,7 & 0,9 & Neossolos Litólicos eutróficos típicos textura argilosa \\
\hline RLe2 & 8,1 & 4,3 & Neossolos Litólicos eutróficos chernossólicos textura média \\
\hline RUd & 10,5 & 5,6 & Neossolos Flúvicos Tb distróficos típicos textura média \\
\hline NVef1 & 16,3 & 8,8 & Nitossolos Vermelhos eutroférricos típicos textura muito argilosa \\
\hline NVef2 & 2,7 & 1,5 & Nitossolos Vermelhos eutroférricos latossólicos textura muito argilosa \\
\hline
\end{tabular}


em peneiras de 2,0 $\mathrm{mm}$ para realização das análises físicas, químicas e mineralógicas. Os grupamentos texturais dos solos foram definidos conforme Embrapa (1999). Pelo método do densímetro (Camargo et al., 1986), determinaram-se os teores de areia total, silte e argila. Teores de $\mathrm{Ca}, \mathrm{Mg}, \mathrm{K}$ e soma de bases (S) foram determinados conforme Raij \& Quaggio (1989).
Matéria orgânica (MO), acidez total e trocável, $\mathrm{pH}$ em água e em $\mathrm{KCl}$, capacidade de troca catiônica (CTC), saturação por bases (V \%) e saturação por alumínio (m \%) foram determinados conforme Embrapa (1997). Os teores de Fe total, Si e Ti foram determinados por meio de ataque sulfúrico, conforme método preconizado pela Embrapa (1997) (Quadro 2).

Quadro 2. Atributos químicos e físicos ${ }^{(1)}$ do horizonte superficial (A) e subsuperficial (B) dos solos encontrados na área de estudo

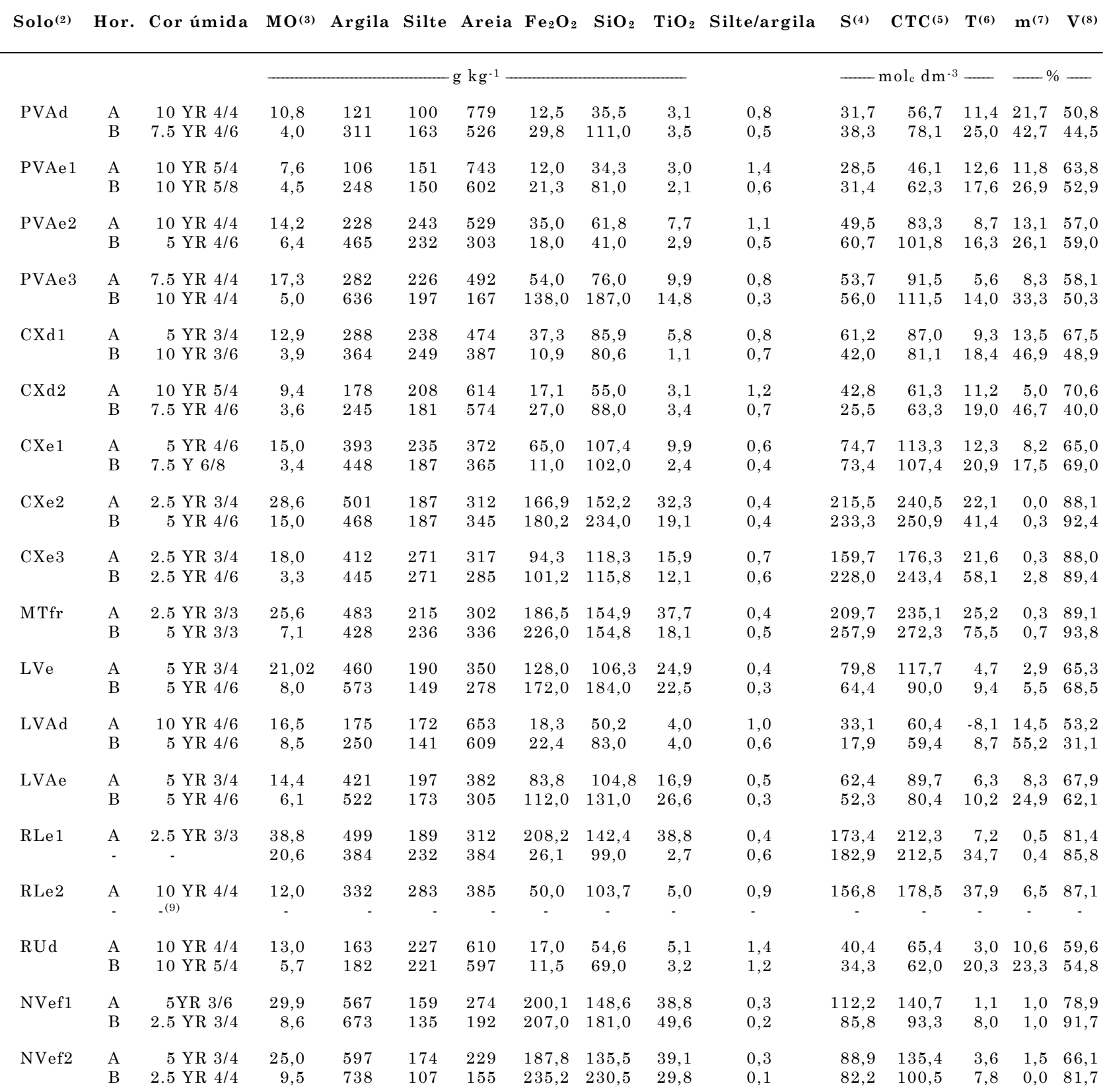

(1) Valores médios dos pontos amostrais da unidade de mapeamento. ${ }^{(2)}$ Ver Quadro 1. ${ }^{(3)}$ MO: Matéria orgânica. ${ }^{(4)}$ S: Soma de bases. ${ }^{(5)}$ CTC: Capacidade de troca catiônica. ${ }^{(6)} \mathrm{T}$ : Atividade da argila. ${ }^{(7)} \mathrm{m}$ : Saturação por alumínio. ${ }^{(8)} \mathrm{V}$ : Saturação por bases. ${ }^{(9)}$ Não determinado. 
Para identificar a resposta espectral dos solos em laboratório, as amostras secas e peneiradas foram devidamente acondicionadas em placas de petri de $9 \mathrm{~cm}$ de diâmetro. Em ambiente devidamente controlado, foram efetuadas leituras radiométricas utilizando-se o espectrorradiômetro IRIS, Infra-Red Intelligent Spectroradiometer (GER, 1996), com resolução espectral de $2 \mathrm{~nm}$ (de 300-1.000 nm) e de $4 \mathrm{~nm}$ (de $1.000-3.000 \mathrm{~nm})$.

A geometria de aquisição dos dados foi: (a) placa padrão branca com $100 \%$ de reflectância calibrada (Labsphere, 1996); (b) distância do alvo ao sensor, $27 \mathrm{~cm}$; (c) distância da fonte ao alvo, $61 \mathrm{~cm}$; (d) fonte de iluminação: lâmpada halógena de $650 \mathrm{~W}$ com refletor parabólico e feixe não colimado ligada em fonte estabilizadora de alta exatidão com entrada de $220 \mathrm{~V}$ e saída regulada em $110 \pm 0,5 \mathrm{~V}$ de tensão nominal e 5,2 ampères.

A geometria seguiu os procedimentos de Valeriano et al. (1995), Galvão et al. (1995), Galvão et al. (1997), Galvão \& Vitorello (1998) e Demattê et al. (1998). Apesar desses autores não terem comentado quanto à altura entre o piso e a fonte luminosa, foi adotada, para este trabalho, a altura de $1,20 \mathrm{~m}$, em face da necessidade de padronização do sistema de iluminação e maior performance do sistema.

Foram realizadas, para cada amostra, três leituras espectrais girando-se a placa de petri cerca de 120 graus entre cada leitura, para que fosse feita a varredura de diferentes pontos da placa, das quais se extraiu a média para ser utilizada nas discussões. Portanto, foi obtido o fator de reflectância bidirecional espectral, que expressa a razão entre o fluxo radiante espectral refletido pela superfície de um corpo e o fluxo radiante espectral refletido por um padrão de referência, sob as mesmas condições de iluminação e geometria de leitura (Nicodemus et al., 1977).

Os dados de reflectância foram tabulados em planilhas eletrônicas e, então, usados para gerar as bandas do TM-Landsat simuladas. Para este fim a média da resposta espectral obtida pelo sensor IRIS foi calculada nos intervalos de onda correspondentes às bandas $1(450-520 \mathrm{~nm}), 2(520-600 \mathrm{~nm}), 3(630$ $690 \mathrm{~nm}), 4(760-900 \mathrm{~nm}), 5(1.150-1.750 \mathrm{~nm})$ e 7 $(2.080-2.350 \mathrm{~nm})$ do TM-Landsat. Juntamente com as bandas, os dados tabulados possibilitaram a construção dos gráficos relativos à relação entre as bandas espectrais.

\section{RESULTADOS E DISCUSSÃO}

\section{Linha do solo calculada pelos dados espectrais obtidos em laboratório}

Como a reflectância foi extraída de amostras de solo, em laboratório, os vários pontos apresentaramse bem próximos à linha do solo (Figura 1). Esses resultados indicam que as amostragens realmente extraíram dados de reflectância apenas do solo, pois a dispersão de pontos do gráfico encontra-se aproximadamente numa faixa ao longo de $45^{\circ} \mathrm{em}$ relação ao eixo $\mathrm{x}$, com valores de $\mathrm{R}^{2}$ próximos ao valor 1,0. Tal fato era esperado, haja vista serem as amostras analisadas em laboratório e não terem nenhuma interferência da vegetação. Os valores de $\mathrm{R}^{2}$ são maiores, quando comparados com os resultados apresentados por Nanni (2000) e Demattê (1995), que utilizaram a linha de solo obtida por meio de imagens orbitais. Outro fato interessante refere-se à pequena influência da MO no comportamento da linha do solo, discordando das observações relatadas por Galvão \& Vitorello (1998).

Para alguns autores, todos os solos podem ser representados por uma única linha "global" do solo. Entretanto, Huete et al. (1984) e Baldy et al. (1986) observaram que linhas do solo específicas (individuais) demonstraram melhor a situação das propriedades óticas dos solos. Baret et al. (1993), avaliando as variações nas linhas do solo e suas características, concluíram que seus resultados também não suportaram a idéia de uma linha global para os solos.

Objetivando demonstrar a diferenciação entre as classes de solos por meio da linha de solo, foi estabelecida, para cada uma, a relação entre as bandas 3 e 4, para as duas camadas (Figuras $2 \mathrm{a}$ e b).

Quando novamente observados em conjunto, estes dados reforçam a idéia de solos sem influência da vegetação, uma vez que foram obtidos em laboratório. Os valores de $\mathrm{R}^{2}$ das equações de tendência, para cada classe de solo, são todos próximos a 1,0, ou seja, sem dispersão dos dados e com alinhamento em uma abscissa imaginária (Quadro 3).

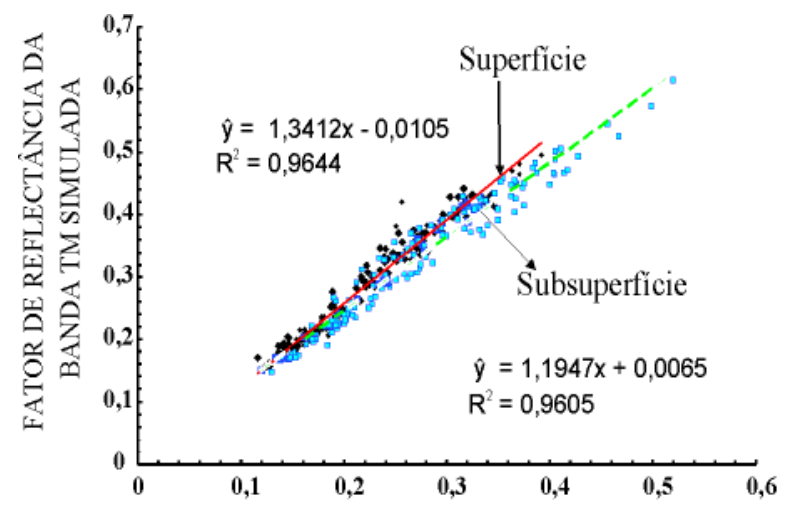

FATOR DF RFFI.FCTÂNCIA DA BANDA TM3 SIMUI.ADA

Figura 1. Linha do solo das amostras de superfície e subsuperfície obtida pela relação da reflectância entre as bandas 3 e 4 simuladas para as faixas do sensor TM, com os dados obtidos em laboratório. 


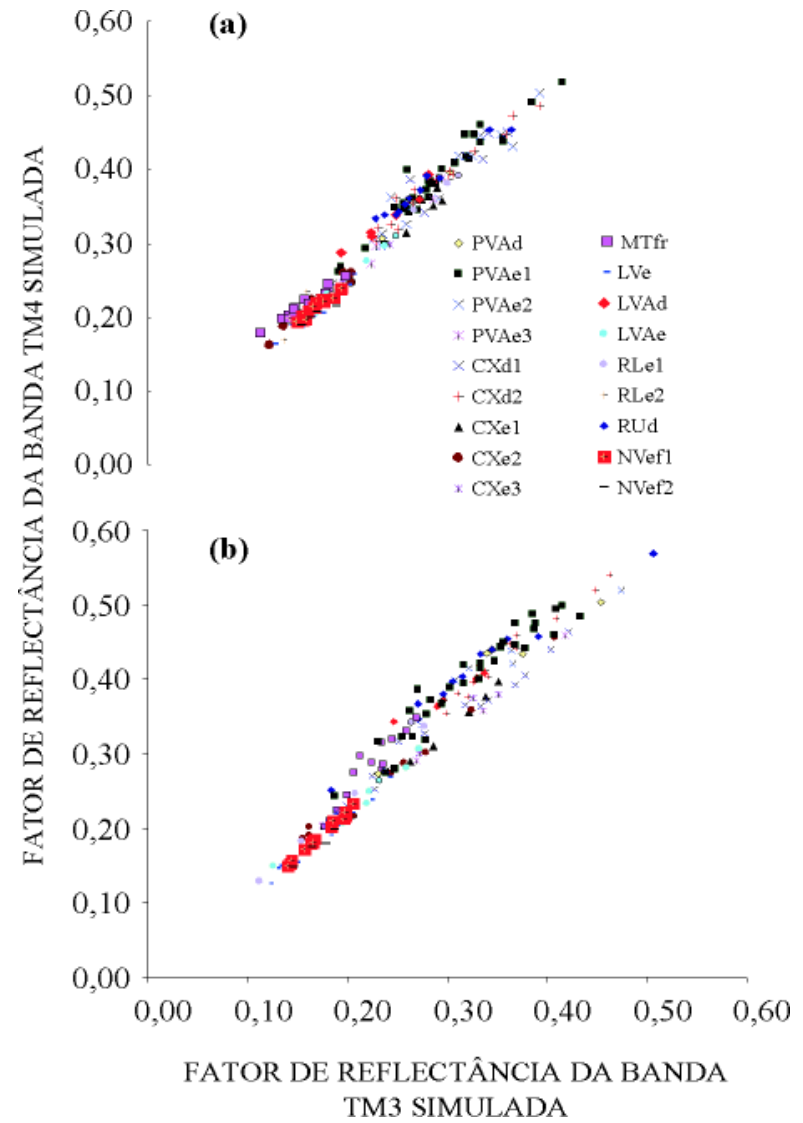

Figura 2. Linha do solo de amostras de solo da camada superficial (a) e subsuperficial (b), para as classes de solos da área de estudo.

Para melhor visualização e discussão das classes com características destoantes, foram escolhidas: a NVef1, RUd, LVe, NVef2, CXd2, MTfr, RLe2 e PVAe1, cujas linhas de solos são apresentadas individualmente (Figura 3).

Observa-se que os solos que, em sua constituição, contêm maiores teores de Fe total, argilosos e com maior albedo, ou seja, NVef1, NVef2, RLe2, LVe e MTfr, apresentaram-se agrupados próximos à interseção dos eixos x, y, confirmando Nanni et al. (2004). Afastados desta interseção, apareceram agrupados os solos com características arenosas, menor albedo e com baixos teores de Fe total (RUd, CXd2 e PVAe1), como também destacado por Galvão \& Vitorello (1998), Demattê (1999) e Dematte et al. (2000). Contudo, conforme observado por Galvão \& Vitorello (1998), os dados não se encontram exatamente dispostos num ângulo de $45^{\circ}$ em relação ao eixo $\mathrm{x}$, tendo, sim, uma ligeira inclinação. No entanto, observa-se a possibilidade de uso da linha do solo como elemento discriminador das classes avaliadas.

Avaliando a distribuição dos dados ao longo da linha, verificou-se que não houve diferenças entre os solos mais ricos em Fe, com exceção da classe RLe2 que não participou da análise por não apresentar
Quadro 3. Classes de solos, equações de tendência linear e $R^{2}$ para a relação entre as bandas 3 e 4 simuladas para o sensor TM, obtidas em laboratório, das amostras de superfície e subsuperfície

\begin{tabular}{|c|c|c|c|}
\hline $\begin{array}{c}\text { Classe de } \\
\text { solo }^{(1)}\end{array}$ & Hor. & Equação de tendência & $\mathbf{R}^{2}$ \\
\hline \multirow[t]{2}{*}{ PVAd } & A & $\hat{y}=1,393 x-0,019$ & 0,97 \\
\hline & B & $\hat{y}=1,0258 x+0,0643$ & 0,94 \\
\hline \multirow[t]{2}{*}{ PVAe1 } & A & $\hat{y}=1,2078 x+0,0339$ & 0,93 \\
\hline & B & $\hat{y}=1,0803 x+0,0618$ & 0,92 \\
\hline \multirow[t]{2}{*}{ PVAe2 } & A & $\hat{y}=1,2325 x+0,0177$ & 0,95 \\
\hline & B & $\hat{y}=1,0035 x+0,0482$ & 0,98 \\
\hline \multirow[t]{2}{*}{ PVAe3 } & A & $\hat{y}=1,4712 \mathrm{x}-0,0494$ & 0,97 \\
\hline & B & $\hat{y}=1,0456 x+0,0277$ & 0,99 \\
\hline \multirow[t]{2}{*}{ CXd1 } & A & $\hat{y}=1,1939 x+0,0307$ & 0,91 \\
\hline & B & $\hat{y}=0,9737 x+0,0858$ & 0,92 \\
\hline \multirow[t]{2}{*}{$\mathrm{CXd} 2$} & A & $\hat{y}=1,1282 \mathrm{x}+0,0521$ & 0,96 \\
\hline & B & $\hat{y}=1,0691 x+0,0593$ & 0,98 \\
\hline \multirow[t]{2}{*}{ CXe1 } & A & $\hat{y}=1,3062 \mathrm{x}-0,0147$ & 0,95 \\
\hline & B & $\hat{y}=1,2367 x-0,027$ & 0,99 \\
\hline \multirow[t]{2}{*}{$\mathrm{CXe} 2$} & A & $\hat{y}=1,1302 x+0,0225$ & 0,95 \\
\hline & B & $\hat{y}=1,0576 x+0,0298$ & 0,99 \\
\hline \multirow[t]{2}{*}{ CXe3 } & A & $\hat{y}=1,0368 x+0,042$ & 0,99 \\
\hline & B & $\hat{y}=1,0668 x+0,0277$ & 0,93 \\
\hline \multirow[t]{2}{*}{ MTfr } & A & $\hat{y}=0,9812 x+0,0535$ & 0,94 \\
\hline & B & $\hat{y}=1,5231 \mathrm{x}-0,0487$ & 0,94 \\
\hline \multirow[t]{2}{*}{$\mathrm{LVe}$} & A & $\hat{y}=1,1873 x+0,0044$ & 0,94 \\
\hline & B & $\hat{y}=1,1219 x+0,0073$ & 0,97 \\
\hline \multirow[t]{2}{*}{ LVAd } & A & $\hat{y}=1,1873 x+0,0044$ & 0,94 \\
\hline & B & $\hat{y}=0,7408 x+0,1675$ & 0,98 \\
\hline \multirow[t]{2}{*}{ LVAe } & A & $\hat{y}=1,2961 x-0,0136$ & 0,98 \\
\hline & B & $\hat{y}=1,0796 x+0,0186$ & 0,98 \\
\hline RLe1 & A & $\hat{y}=0,7548 x+0,1496$ & 0,99 \\
\hline RLe2 & A & $\hat{y}=1,7659 x-0,0707$ & 0,99 \\
\hline \multirow[t]{2}{*}{ RUd } & A & $\hat{y}=1,0545 x+0,0798$ & 0,96 \\
\hline & B & $\hat{y}=0,9682 x+0,1052$ & 0,97 \\
\hline \multirow[t]{2}{*}{ NVef1 } & A & $\hat{y}=1,0258 x+0,0344$ & 0,92 \\
\hline & B & $\hat{y}=1,194 x-0,0034$ & 0,97 \\
\hline \multirow[t]{2}{*}{ NVef2 } & A & $\hat{y}=0,7379 x+0,0706$ & 0,94 \\
\hline & B & $\hat{y}=1,118 x-0,0003$ & 0,93 \\
\hline
\end{tabular}

(1) Valores médios dos pontos amostrais da unidade de mapeamento. 


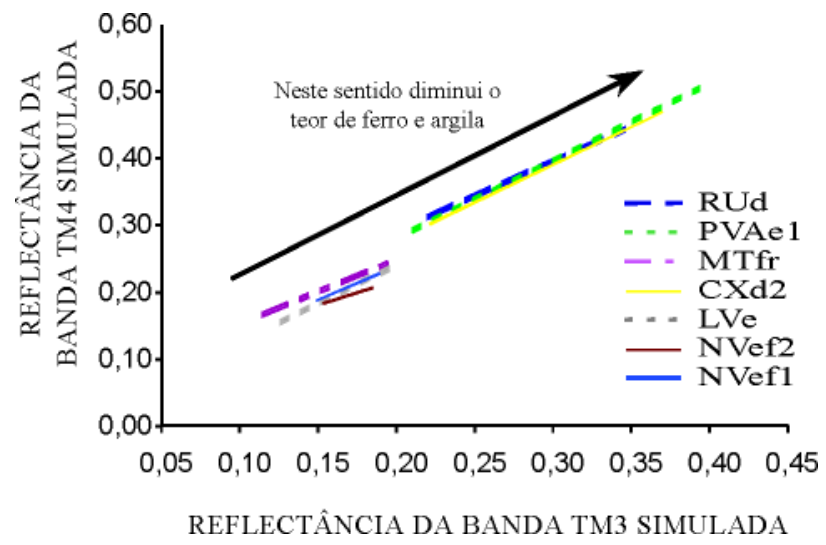

Figura 3. Linha do solo de amostras da camada subsuperficial, para algumas das classes de solos da área de estudo e o seu deslocamento em relação aos teores de ferro e argila nas amostras analisadas.

horizonte subsuperficial. Sabe-se que a reflectância ao longo da linha do solo é influenciada principalmente pela MO, óxidos de Fe e umidade (Galvão \& Vitorello, 1998). Como a umidade foi homogeneizada em todas as amostras, levou-se em consideração que os teores de $\mathrm{MO}$ e teores de Fe total foram próximos, com coeficiente de variação de $12,6 \%$ e amplitudes de 7,1 a $9,5 \mathrm{~g} \mathrm{~kg}^{-1}$, e de $13,3 \%$ e amplitudes de 172 a $135,2 \mathrm{~g} \mathrm{~kg}^{-1}$ respectivamente. Explica-se, assim, o comportamento de seus posicionamentos na linha, como estabelecido por Galvão \& Vitorello (1998).

Como foram determinados teores de Fe total, maior atenção deverá ser dada em novos estudos não só sobre o comportamento da linha do solo para diferentes formas de óxidos encontrados (amorfo e cristalino), mas também sobre a fragmentação da MO, uma vez que esses compostos podem interferir na resposta espectral dentro do intervalo avaliado, como destacado por Demattê et al. (2003).

Contudo, a inclinação foi variada para cada classe. Observou-se que a inclinação apresentada pela classe RLe2 foi ligeiramente maior que a das demais classes agrupadas. A classe NVef2 foi a que apresentou menor inclinação para este grupo. Para o grupo dos solos arenosos, a inclinação, no entanto, foi muito semelhante. Um mesmo solo, com diferentes texturas, parece apresentar inclinações semelhantes, com ligeira diferença na intensidade, como também observado por Demattê et al. (2000). Este ponto, entretanto, carece de maiores investigações, uma vez que a associação de fatores deve influenciar a inclinação da linha e não apenas a textura isoladamente.

Em relação à intensidade, quando se analisaram solos com amplitude textural maior, como as classes RUd e os NVef, as diferenças foram marcantes. Aliás, observou-se que os solos com textura argilosa e maiores teores de Fe têm os pontos concentrados em reflectâncias mais baixas, mas com maior dispersão ao longo da linha do solo que os arenosos. Por sinal, as correlações dos solos arenosos são um pouco melhores que as dos argilosos, ao contrário dos dados observados por Demattê (1999).

Como se nota, os dados indicaram que a linha do solo foi influenciada pelas propriedades do solo, como óxido de Fe, textura e muito pouco pela MO. Os dois primeiros elementos concordam com os dados descritos por Galvão \& Vitorello (1998), discordando apenas no atributo MO. As variações nos valores dos diferentes solos e a tendência de agrupamento das amostras referentes ao mesmo solo, ao longo da curva, levam a crer que o solo deve dispor de uma linha individual, concordando com observações de Huete et al. (1984) e Baret et al. (1993).

\section{CONCLUSÕES}

1. A avaliação da "linha de solo" estabelecida pela reflectância dos solos obtida em laboratório apresentou-se como um método auxiliar na discriminação de classes de solos. Os dados evidenciam que cada solo apresenta uma linha individual e característica.

2. A avaliação dos dados na linha do solo demonstrou que solos arenosos e com menores teores de ferro discriminaram-se dos demais, apresentando valores de reflectância mais elevados.

3. Tanto as amostras de superfície como as de subsuperfície apresentaram comportamento semelhante em relação à linha do solo avaliada graças à ausência de material vegetal.

4. A matéria orgânica encontrada nos solos avaliados não constitui fator determinante no comportamento espectral em relação à linha do solo.

\section{AGRADECIMENTOS}

À Fundação de Amparo à Pesquisa do Estado de São Paulo-FAPESP, pelo auxílio financeiro (Proc. $n$. 983516-98), à CAPES, pela bolsa do primeiro autor, e ao CNPq, pela bolsa pesquisador para o co-autor (Proc. n. 300371/96-9).

\section{LITERATURA CITADA}

BALDY, C.; BARET, F. \& TRIGUI, A. Analyse des comportements spectraux dans 1'olivette de fax. Agronomie., 6:941-948, 1986.

BARET, F.; JACQUEMOUND, S. \& HANOQ, J.F. The soil line concept in remote sensing. Remote Sens. Environ., $7: 1-18,1993$.

CAMARGO, A.O.; MONIZ, A.C.; JORGE, J.A. \& VALADARES, J.M. Métodos de análise química, mineralógica e física de solos do IAC. Campinas, Instituto Agronômico, 1986. 94 p. 
DEMATTÊ, J.A.M. Relações entre dados espectrais e características físicas, químicas e mineralógicas de solos desenvolvidos de rochas eruptivas. Piracicaba, Escola Superior de Agricultura "Luiz de Queiroz", 1995. 265p. (Tese de Doutorado)

DEMATTÊ, J.A.M.; SOUSA, A.A. \& NANNI, M.R. Avaliação espectral de amostras de solo e argilo-minerais em função de diferentes níveis de hidratação. In: SIMPÓSIO BRASILEIRO DE SENSORIAMENTO REMOTO, 9., Santos, 1998. Anais. Santos, INPE/SELPER, 1998. CDROM

DEMATTÊ, J.A.M. Reflectância espectral de solos. Piracicaba, Escola Superior de Agricultura "Luiz de Queiroz", 1999. 451p. (Tese de Livre Docência)

DEMATTÊ, J.A.M.; HUETE, A.R.; FERREIRA, L.G.; ALVES, M.C.; NANNI, M.R. \& CERRI, C.E. Evaluation of tropical soils throught ground and orbital sensors. In: INTERNATIONAL CONFERENCE GEOESPACIAL INFORMATION IN AGRICULTURE AND FORESTRY, 2., 2000. Proceedings. Lake Buena Vista, Florida, ERIM, 2000. p.34-41.

DEMATTÊ, J.A.M. \& NANNI, M.R. Weathering sequence of soils developed from basalt as evaluated by laboratory (IRIS), airborne (AVIRIS) and orbital (TM) sensors. Inter. J. Remote Sens., 24:4715-4738, 2003.

DEMATTÊ, J.A.M.; EPIPHANIO, J.C.N. \& FORMAGGIO, A.R. Influência da matéria orgânica e formas de ferro na reflectância dos solos tropicais. Bragantia, 62:451-464, 2003.

EMPRESA BRASILEIRA DE PESQUISA AGROPECUÁRIA EMBRAPA. Manual de métodos de análise de solo. 2.ed. Rio de Janeiro, Ministério da Agricultura, 1997. 212p.

EMPRESA BRASILEIRA DE PESQUISA AGROPECUÁRIA EMBRAPA. Centro Nacional de Pesquisa dos Solos. Sistema Brasileiro de Classificação de Solos. Rio de Janeiro, 1999. 412p.

FUKUHARA, M.; HAYASIU, S. \& YASUDA, Y. Extraction of soil information from vegetated areas. In: MACHINE PROCESSING OF THE REMOTELY SENSED DATA, 3., West Lafayette, 1979. Proceedings. West Lafayette, Purdue University, 1979. p.242-252.

GALVÃO, L.S.; VITORELLO, Í. \& PARADELLA, W. Spectrodadiometric discrimination of laterites with pricipal components analysis and additive modeling. Remote Sens. Environ., 53:70-75, 1995.

GALVÃO, L.S.; VITORELLO, Í. \& FORMAGGIO, A.R. Relationships of spectral reflectance and color among surface and subsurface horizons of tropical soil profíles. Remote. Sens. Environ., 61:24-33, 1997.

GALVÃO, L.S. \& VITORELLO, Í. Variability of laboratory measured soil lines of soil from southeastern Brazil Remote Sens. Environ., 6:166-181, 1998.
GALVÃO, L.S.; VITORELLO, Í. \& ALMEIDA FILHO, R. Effects of bands positioning and bandwidth on NDVI measurement of tropical savannas. Remote Sens. Environ., 67:181-193, 1999

GEOPHYSICAL ENVIRONMENTAL RESEARCH CORP. GER. Mark V Dual Field of View IRIS Manual. Version 1.3. New York, Milbook, 1996. 63p.

HUETE, A.R.; POST, D.F. \& JACKSON, R.D. Soil spectra effects on 4 -space vegetation discrimination. Remote Sens. Environ., 15:155-165, 1984.

HUETE, A.R. Soil influences in remotely sensed vegetationcanopy spectra. In: ASRAR, G., ed. Theory and application of optical remote sensing. New York, Wiley Interscience, 1989. p.107-141.

INSTITUTO DE PESQUISAS TECNOLÓGICAS - IPT Divisão de minas e geologia aplicada. Mapa geológico do Estado de São Paulo. São Paulo, 1981. Escala 1:1000.000.

INSTITUTO NACIONAL DE PESQUISAS ESPACIAIS - INPE. Tutorial Spring: Spring básico. São José dos Campos, 1999.

JACKSON, R.D. Spectral indices in space. Remote Sens. Environ., 13:409-421, 1983

KAUTH, R.J. \& THOMAS, J.S. The tasseled capa graphic description of the spectral temporal development of agricultura crops as seen by Landsat. In: MACHINE PROCESSING OF REMOTELY SENSED DATA, 1., West Lafayette 1976. Proceedings. West Lafayette, Purdue University, 1976. p.242-252.

LATZ, R.A.; WEISMILLER, G.E.\& van SCOTOC, BAUMGARDNER, M.F. Characteristic variations in spectral reflectance of selected eroded Alfisols. Soil Sci. Soc. Am. J., 45:1130-1134, 1984.

LABSPHERE, REFLECTANCE CALIBRATION LABORATORY. Spectral reflectance target calibrated from 0.25-2.5 $\mu \mathrm{m}$ reported in $0.050 \mu \mathrm{m}$ intervals. Sutton, 1996. 5p.

NANNI, M.R.; DEMATTÊ, J.A.M. \& FIORIO, P.R. Análise discriminante dos solos por meio da resposta espectral no nível terrestre. Pesq. Agropec. Bras., 39:995-1006, 2004.

NANNI, M.R. Dados radiométricos obtidos em laboratório e no nível orbital na caracterização e mapeamento dos solos. Piracicaba, Escola Superior de Agricultura "Luiz de Queiroz", 2000. 365p. (Tese de Doutorado)

NICODEMUS, F.E., RICHMOND, J.C.; HSIA, J.J.; GINSBERG, I.W. \& LIMPERIS, T. Geometrical considerations and nomenclature for reflectance. Washington, U.S. Department of Commerce, 1977. 52p. (Monograph)

RAIJ, B. van \& QUAGGIO, J.A. Métodos de análise de solo para fins de fertilidade. Campinas, Instituto Agronômico de Campinas, 1989. 40p.

VALERIANO, M.M.; EPIPHANIO, J.C.N.; FORMAGGIO, A.R. \& OLIVEIRA, J.B. Bi-directional reflectance factor of 14 soil classes from Brazil. Inter. J. Remote Sens., 16:113128,1995 . 